Supporting Information for

\title{
Rapid Detection of COVID-19 Using MALDI-TOF-based Serum Peptidome Profiling
}

Ling Yan, ${ }^{1, \#}$ Jia Yi, ${ }^{2, \#}$ Changwu Huang, ${ }^{3, \# ~ J i a n ~ Z h a n g, ~}{ }^{4, \#}$ Shuhui Fu, ${ }^{5}$ Zhijie Li, ${ }^{1}$ Qian Lyu, Yuan $\mathrm{Xu},{ }^{1}$ Kun Wang, ${ }^{1}$ Huan Yang, ${ }^{1}$ Qingwei Ma,${ }^{5}$ Xiaoping Cui, ${ }^{6}$ Liang Qiao, ${ }^{2, *}$ Wei Sun, ${ }^{4, *}$ and $\mathrm{Pu} \mathrm{Liao}{ }^{1, *}$

1: Department of clinical laboratory, Chongqing General Hospital, Chongqing 400014, China

2: Department of chemistry, Fudan University, Shanghai 200438, China

3: Department of clinical laboratory, Renji Hospital, University of Chinese Academy of Sciences, Chongqing, China

4: State Key Laboratory of Proteomics, Beijing Proteome Research Center, National Center for Protein Sciences (Beijing), Beijing Insti-tute of Lifeomics, Beijing 102206, China

5: Bioyong Technologics Inc., Beijing 100176, China

6: Department of clinical laboratory, Sanxia Hospital Affiliated to Chongqing University, Chongqing 404000, China

\# Equal contribution.

*Email: liaopu@sina.com (P. Liao); sunway21cn@126.com (W. Sun); liang_qiao@fudan.edu.cn (L. Qiao).

\section{Table of Contents}

Text S1. Supplementary Methods.

Figure S1. The representative MALDI-TOF mass spectra of samples from COVID-19 patients and control participants.

Figure S2. Relative intensity comparison of the 25 features between controls $(n=101)$ and COVID-19 patients $(\mathrm{n}=97)$ in the training cohort.

Figure S3. The Gene Ontology (GO) processes enriched by Metascape.

Table S1. Demographics of COVID-19 patients and controls.

Table S2. Intensities of feature peaks in the training cohort.

Table S3. Intensities of feature peaks in the test cohort.

Table S4. Annotation of the selected features. 


\section{Text S1: SUPPLEMENTARY METHODS}

MALDI-TOF Data Processing. MALDI-TOF raw data were converted to txt and then processed with $\mathrm{R}$ packages MALDIquant and MALDIquantForeign. ${ }^{1}$ The mass range was adjusted to 5 to $30 \mathrm{kDa}$. Then, the square root transformation was applied, followed by Savitzky-Golay smoothing, ${ }^{2}$ and SNIP baseline correction. ${ }^{3}$ The mass value alignment was performed with the warpMassSpectra command. Peak detection was conducted with a signalto-noise ratio of 3 and a half window size of 20. Peaks were binned with the binPeaks command with a tolerance of 0.002 . Peak filtration was applied with the filterPeaks command to keep the peaks with frequency $\geq 25 \%$ in all spectra of a group (COVID-19 or control). Finally, the obtained matrices were used for the following analysis.

Statistical Analysis. The matrix of peak intensities was subjected to quantile normalization ${ }^{4}$ with an $\mathrm{R}$ package limma after $\log 2$ transformation. Missing values were imputed with the minimum value across all samples. The data of COVID-19 patients and controls were randomly split into training and test cohorts with an allocation of 2:1, corresponding to 198 and 100 samples, respectively.

The partial least squares-discriminant analysis (PLS-DA) was performed using Metaboanalyst $4.0^{5}$ (McGill University, Montreal, Canada, https://www.metaboanalyst.ca/). The variable importance for the projection (VIP) identified by PLS-DA showed the contribution of each feature, and the peaks with top 20 VIP scores were selected as features of COVID19.

A two-tailed t-test was used to identify the differentially expressed peaks between COVID-19 and control samples on the training dataset. The $\mathrm{P}$ values were corrected by the Benjamini-Hochberg algorithm. Peaks with adjusted $\mathrm{P}<0.05$ and fold change $>1.5$ were used for further feature selection with least absolute shrinkage and selection operator $(\text { LASSO })^{6}$ regression. For the LASSO selection, $80 \%$ samples of the training dataset were selected randomly with replacement 200 times $^{7}$ and the peaks with top 20 repeat occurrence frequencies were selected as features of COVID-19. The parameter lambda was determined based on a 10-fold cross-validation. LASSO analysis was conducted in R version 3.5.3 with the glmnet ${ }^{8}$ package. 
Recursive feature elimination with cross-validation (RFECV) was performed using the sklearn.feature_selection.RFECV package in Jupyter Notebook 5.7.9 and the estimator of parameters was logical regression and the $\mathrm{CV}$ of parameters was $10 .^{9}$

Machine Learning. Logistic regression (LR) was performed using the sklearn.linear_model.LogisticRegression package in Jupyter Notebook 5.7.9 and the class_weight of parameters was balanced. ${ }^{10}$ Support vector machine (SVM) was performed using the sklearn.svm.SVC package in Jupyter Notebook 5.7.9 and the probability of parameters was true and the kernel of parameters was linear. ${ }^{11}$ Naive bayes (NB) was performed using the sklearn.naive_bayes. GaussianNB package in Jupyter Notebook 5.7.9. with default parameters. $^{12}$ Decision tree (DT) was performed using the sklearn.tree.DecisionTreeClassifier package in Jupyter Notebook 5.7.9. and the random_state of parameters was $42 .{ }^{13}$ Random forest (RF) was performed using the sklearn.enseble. RandomForestClassifier package in Jupyter Notebook 5.7.9 and the random_state of parameters was $42 .{ }^{14}$ Gradient boosting decision tree (GBDT) was performed using the sklearn.enseble. GradientBoostingClassifier package in Jupyter Notebook 5.7.9 and the random_state of parameters was 42. ${ }^{15}, 16$ AdaBoost was performed using the sklearn.enseble.AdaBoostClassifier package in Jupyter Notebook 5.7.9 with default parameters. ${ }^{17} \mathrm{~K}$-nearest neighbors $(\mathrm{KNN})$ was performed using the sklearn.neighbors. KNeighborsClassifier package in Jupyter Notebook 5.7.9 with default parameters. ${ }^{18}$

Calculation of Accuracy, Sensitivity, Specificity, Precision and F1. Accuracy, sensitivity, specificity, precision and F1 were used to evaluate the performance of the models. Accuracy is defined as a ratio between the correctly classified samples to the total number of samples as indicated in Eq. (1), ${ }^{19}$

$$
\text { Accuracy }=\frac{T P+T N}{T P+T N+F P+F N}
$$

where TP stands for true positive, TN for true negative, FP for false positive and FN for false negative. Sensitivity represents the correctly classified positive samples to the total number of positive samples, Eq. (2), ${ }^{19}$ 


$$
\text { Sensitivity }=\frac{T P}{T P+F N}
$$

Specificity represents the correctly classified negative samples to the total number of negative samples as indicated in Eq. (3), ${ }^{19}$

$$
\text { Specificity }=\frac{T N}{F P+T N}
$$

Precision represents the proportion of positive samples that were correctly classified to the total number of predicted positive samples as indicated in Eq. (4), ${ }^{19}$

$$
\text { Precision }=\frac{T P}{F P+T P}
$$

F1-score represents the harmonic mean of precision and sensitivity as indicated in Eq. (5), ${ }^{19}$

$$
F 1-\text { score }=\frac{2 T P}{2 T P+F P+F N}
$$

The F1-score is ranged from zero to one, and high values of F1-score indicate high classification performance.

Proteomic Analysis. The sera from 4 COVID-19 patients (VSample 67, 81, 112, 140) and 3 healthy controls (Sample 36, 41, 145) were selected for proteomic analysis. The MALDITOF mass spectra of the 7 samples contained all the 25 feature peaks. After $56^{\circ} \mathrm{C}$ sterilization in $40 \mathrm{mM}$ dithiothreitol for $30 \mathrm{~min}, 1 \mu \mathrm{L}$ of each serum sample was separated by $16.5 \%$ Tris-tricine-SDS-PAGE gel. Then the gel band ranged 5-40 kDa of each sample was cut and destained. After dithiothreitol reduction and iodoacetamide alkylation, the samples were digested by trypsin (sequencing grade, Promega, USA) overnight at $37{ }^{\circ} \mathrm{C}$. Peptide extracts were analyzed by a Q Exactive mass spectrometer (Thermo Fisher Scientific, San Jose, USA) coupled with a nanoflow HPLC instrument (UltiMate 3000 nano, Thermo Fisher Scientific, USA). Each full MS scan was followed by data-dependent acquisition (DDA) MS/MS scans on the 15 most intensive precursor ions.

The LC-MS/MS raw data were analyzed using PEAKS Studio (version X+, Bioinformatics Solutions Inc., Waterloo, Canada). PEAKS DB was set up to search against the Homo sapiens database downloaded from UniProtKB (ver. 202005, 20412 entries), with trypsin as enzyme, and maximum missed cleavages as 2 . The fragment ion mass tolerance was set as $0.02 \mathrm{Da}$ and the parental ion tolerance was set as $7 \mathrm{ppm}$. Carbamidomethylation (C) was 
specified as a fixed modification. Oxidation (M) and acetylation (K) were specified as variable modifications. The false discovery rate (FDR) cut-off on both peptide and protein level was $1 \%$ by using a target-decoy strategy. Proteins were identified based on at least one unique peptide, and quantified based on the top-3 peptides using the extracted MS1 ion current signal with total ion current (TIC) normalization.

Annotation of the Feature Peaks. Matching between the 25 MALDI-TOF mass spectra feature peaks and the proteomic analysis results was performed under the criteria: the molecular weight of an identified protein/protein fragment was consistent to $\mathrm{m} / \mathrm{z}$ of the MALDI-TOF mass peak within a tolerance of $2000 \mathrm{ppm}$; only the charge state of $1+$ was considered for MALDI-TOF; the quantitative variation of the protein/protein fragment between the COVID-19 and the non-COVID-19 groups by LC-MS/MS shows the same trend as that of the matched MALDI-TOF MS peak. If more than one protein/protein fragment was matched to the feature peak under the given criteria, the one with the smallest mass difference was chosen as the identification result. The top Gene Ontology (GO) analysis of the identified feature peaks was performed using Metascape web-based platform. ${ }^{20}$ 


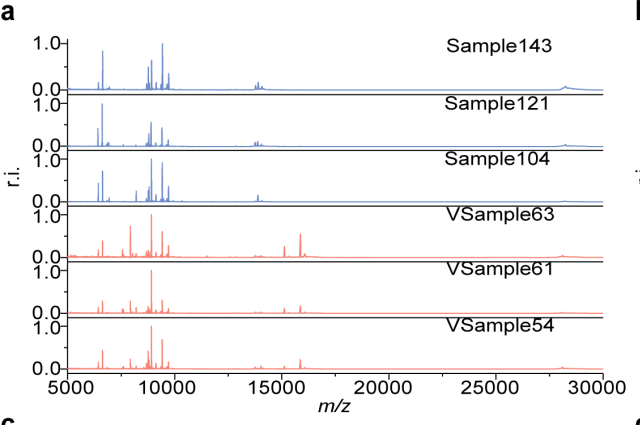

c

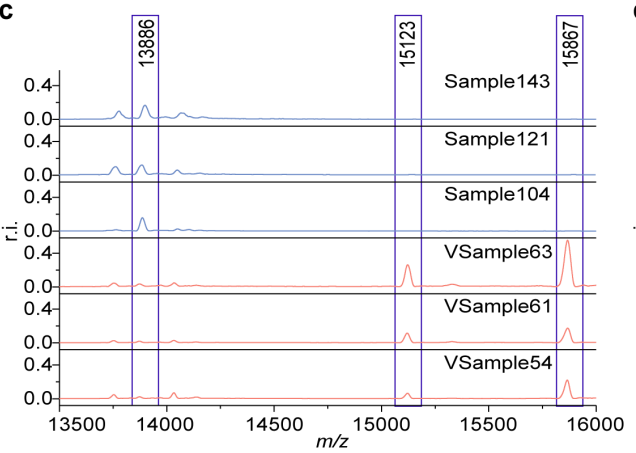

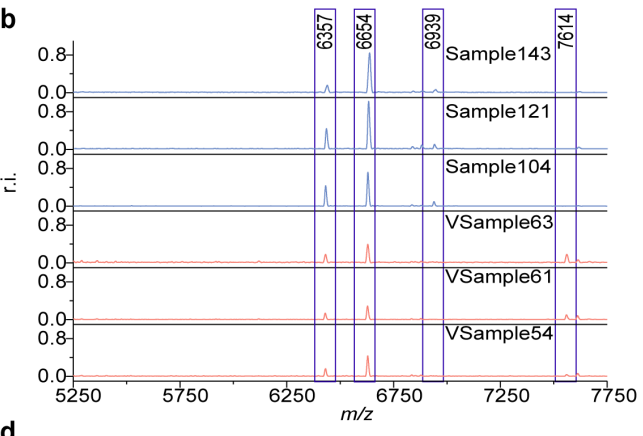

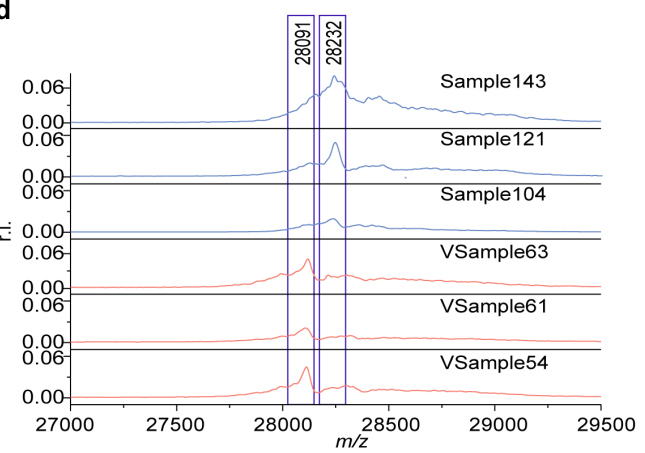

Figure S1. The representative MALDI-TOF mass spectra of samples from COVID-19 patients and control participants. a) MALDI-TOF mass spectra of serum samples from controls (Sample) and COVID-19 patients (VSample). b-d) Partial enlarged view of a). 


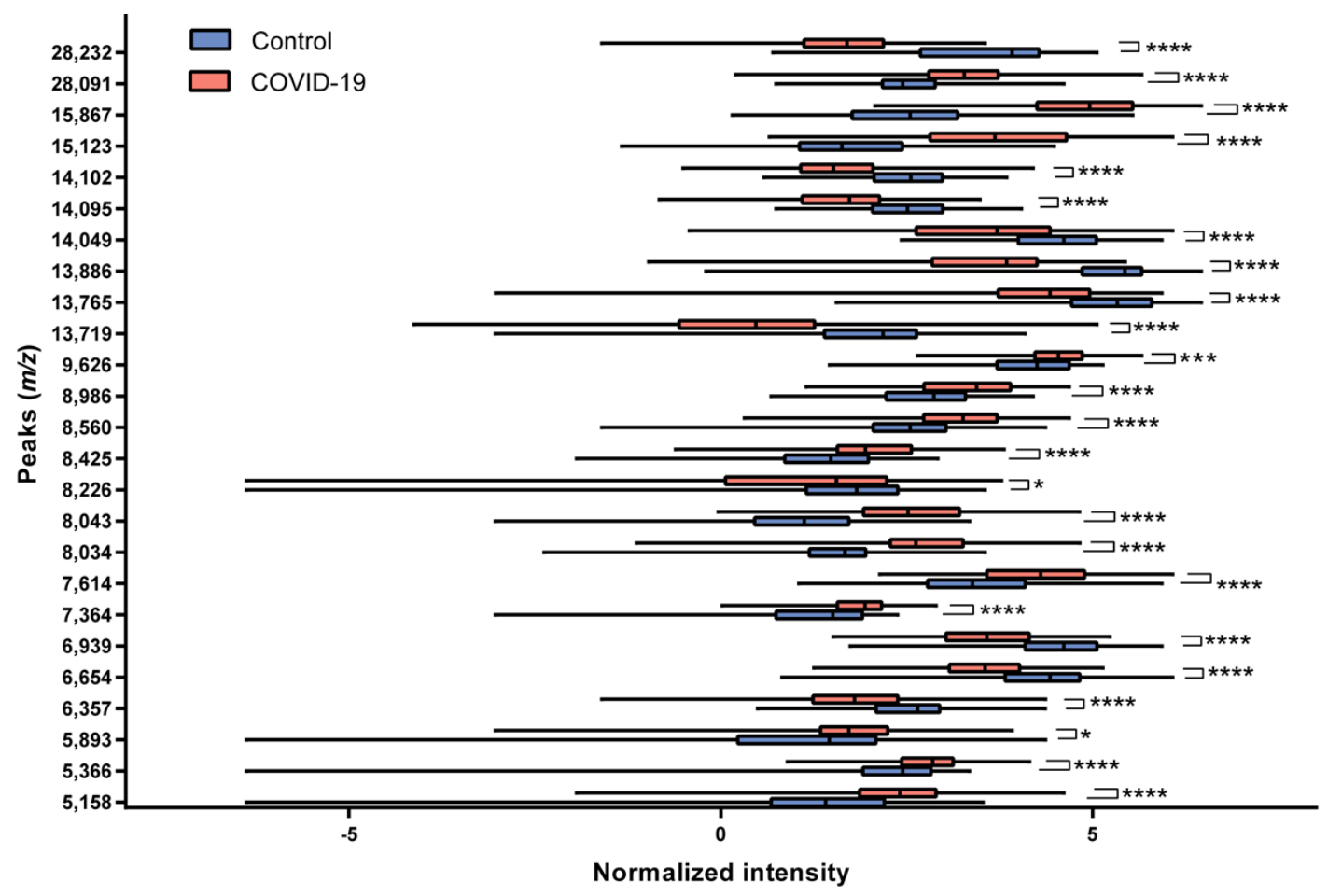

Figure S2. Relative intensity comparison of the 25 features between controls $(\mathrm{n}=101)$ and COVID-19 patients $(\mathrm{n}=97)$ in the training cohort. *: $\mathrm{p}<0.05$; ***: $\mathrm{p}<0.001 ; * * * * \mathrm{p}<$ 0.0001 . P-value is calculated by the Wilcoxon rank-sum test. 


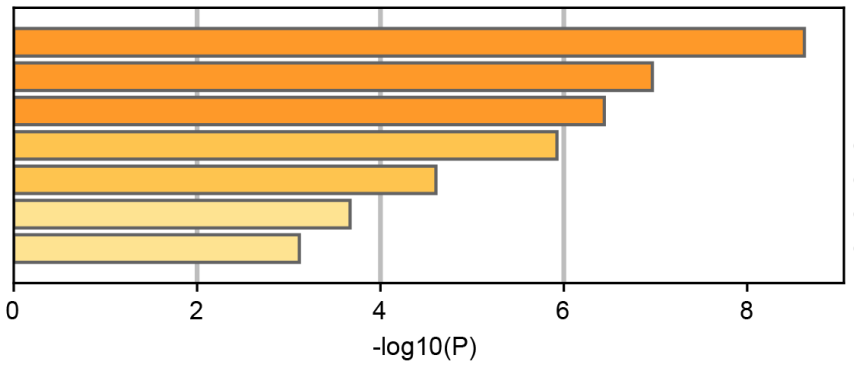

R-HSA-977225: Amyloid fiber formation

R-HSA-6798695: Neutrophil degranulation

R-HSA-9635486: Infection with Mycobacterium tuberculosis

GO:0006959: humoral immune response

GO:0006898: receptor-mediated endocytosis

GO:0002526: acute inflammatory response

GO:0043405: regulation of MAP kinase activity

Figure S3. The Gene Ontology (GO) processes enriched by Metascape ${ }^{20}$ involving the 19 identified features. 
Table S1. Demographics of COVID-19 patients and the control group.

\begin{tabular}{|c|c|c|c|c|c|c|c|c|}
\hline \multirow[b]{2}{*}{ Variables } & \multicolumn{2}{|c|}{ Healthy Control $(\mathrm{N}=46)$} & \multicolumn{2}{|c|}{ Fever and cough $(\mathrm{N}=73)$} & \multicolumn{2}{|c|}{ Tuberculosis $(\mathrm{N}=33)$} & \multicolumn{2}{|c|}{ COVID-19 $(\mathrm{N}=146)$} \\
\hline & $\begin{array}{l}\text { Training } \\
\text { group } \\
(\mathrm{N}=34)\end{array}$ & $\begin{array}{l}\text { Test group } \\
(\mathrm{N}=12)\end{array}$ & $\begin{array}{l}\text { Training } \\
\text { group } \\
(\mathrm{N}=48)\end{array}$ & $\begin{array}{l}\text { Test group } \\
(\mathrm{N}=25)\end{array}$ & $\begin{array}{l}\text { Training } \\
\text { group } \\
(\mathrm{N}=19)\end{array}$ & $\begin{array}{l}\text { Test group } \\
(\mathrm{N}=14)\end{array}$ & $\begin{array}{l}\text { Training } \\
\text { group } \\
(\mathrm{N}=97)\end{array}$ & $\begin{array}{l}\text { Test group } \\
(\mathrm{N}=49)\end{array}$ \\
\hline \multicolumn{9}{|l|}{ Sex $=$ no. $(\%)$} \\
\hline Male & $17(50.0)$ & $8(66.7)$ & $34(70.8)$ & $18(72.0)$ & $14(73.7)$ & $9(64.3)$ & $55(56.7)$ & $28(57.1)$ \\
\hline Female & $17(50.0)$ & $4(33.3)$ & $14(29.2)$ & $7(28.0)$ & $5(26.3)$ & $5(35.7)$ & $42(43.3)$ & $21(42.9)$ \\
\hline \multicolumn{9}{|l|}{ Age-year } \\
\hline Mean \pm SD. & $32.4 \pm 11.4$ & $29.6 \pm 10.2$ & $74.2 \pm 14.4$ & $70.6 \pm 17.6$ & $48.8 \pm 17.1$ & $46.5 \pm 16.3$ & $46.6 \pm 14.9$ & $47.2 \pm 15.4$ \\
\hline Range & $21 \sim 56$ & $20 \sim 51$ & $33 \sim 94$ & $19 \sim 91$ & $25 \sim 73$ & $24 \sim 72$ & $5 \sim 82$ & $20 \sim 80$ \\
\hline \multicolumn{9}{|c|}{ Days from Onset to Sampling } \\
\hline Mean \pm SD. & & & & & & & $14.2 \pm 4.9$ & $11.9 \pm 4.3$ \\
\hline Median(IQR) & & & & & & & 14 & 12 \\
\hline Range & & & & & & & $3 \sim 28$ & $3 \sim 21$ \\
\hline \multicolumn{9}{|l|}{ Symptoms-no.(\%) } \\
\hline Fever & & & $7(14.6)$ & $2(8.0)$ & $0(0.0)$ & $0(0.0)$ & $70(72.2)$ & $32(65.3)$ \\
\hline Fatigue & & & $2(4.2)$ & $1(4.0)$ & $0(0.0)$ & $0(0.0)$ & $17(17.5)$ & $12(24.5)$ \\
\hline Dyspnea & & & $17(35.4)$ & $11(44.0)$ & $0(0.0)$ & $0(0.0)$ & $11(11.3)$ & $4(8.2)$ \\
\hline \multicolumn{9}{|l|}{ Chest CT-no.(\%) } \\
\hline $\begin{array}{l}\text { Involvement of chest } \\
\text { radiographs }\end{array}$ & & & $37(77.1)$ & $13(52.0)$ & $9(47.4)$ & $6(42.9)$ & $95(97.9)$ & $47(95.9)$ \\
\hline
\end{tabular}


Table S2. Intensities of feature peaks in the training cohort.

\begin{tabular}{|c|c|c|c|c|c|c|}
\hline \multirow{2}{*}{$m / z$} & \multicolumn{2}{|c|}{ COVID-19 group } & \multicolumn{2}{|c|}{ Control group } & \multirow{2}{*}{ Raw p-value } & \multirow{2}{*}{$\begin{array}{l}\text { Adjusted } \\
\text { p-value* }\end{array}$} \\
\hline & Median (IQR) & $Q_{1}-Q_{3}$ & Median (IQR) & $Q_{1}-Q_{3}$ & & \\
\hline 5158 & $2.41(0.97)$ & $1.92-2.89$ & $1.41(1.50)$ & $0.68-2.18$ & $1.01 \mathrm{E}-09$ & $1.68 \mathrm{E}-09$ \\
\hline 5366 & $2.85(0.69)$ & $2.44-3.12$ & $2.44(0.88)$ & $1.92-2.80$ & $5.08 \mathrm{E}-06$ & $5.77 \mathrm{E}-06$ \\
\hline 5893 & $1.72(0.87)$ & $1.35-2.22$ & $1.46(1.71)$ & $0.35-2.06$ & $1.30 \mathrm{E}-02$ & $1.30 \mathrm{E}-02$ \\
\hline 6357 & $1.80(1.12)$ & $1.25-2.37$ & $2.64(0.85)$ & $2.09-2.94$ & 2.73E-09 & 4.02E-09 \\
\hline 6654 & $3.55(0.89)$ & $3.10-3.99$ & $4.42(0.98)$ & $3.84-4.82$ & $3.81 \mathrm{E}-12$ & $8.65 \mathrm{E}-12$ \\
\hline 6939 & $3.58(1.10)$ & $3.05-4.14$ & $4.61(0.96)$ & $4.09-5.06$ & $4.75 \mathrm{E}-14$ & $1.48 \mathrm{E}-13$ \\
\hline 7364 & $1.94(0.57)$ & $1.58-2.15$ & $1.50(1.14)$ & $0.74-1.88$ & 5.49E-08 & 7.63E-08 \\
\hline 7614 & $4.30(1.31)$ & $3.58-4.89$ & $3.38(1.31)$ & $2.78-4.09$ & $9.06 \mathrm{E}-08$ & $1.19 \mathrm{E}-07$ \\
\hline 8034 & $2.62(0.96)$ & $2.29-3.25$ & $1.67(0.73)$ & $1.21-1.94$ & $2.06 \mathrm{E}-18$ & $1.03 \mathrm{E}-17$ \\
\hline 8043 & $2.52(1.27)$ & $1.92-3.19$ & $1.12(1.25)$ & $0.47-1.72$ & $7.98 \mathrm{E}-22$ & $6.65 \mathrm{E}-21$ \\
\hline 8226 & $1.55(2.12)$ & $0.11-2.23$ & $1.83(1.17)$ & $1.20-2.37$ & $1.10 \mathrm{E}-02$ & $1.14 \mathrm{E}-02$ \\
\hline 8425 & $1.94(0.95)$ & $1.60-2.55$ & $1.48(1.12)$ & $0.86-1.98$ & $6.46 \mathrm{E}-07$ & $8.08 \mathrm{E}-07$ \\
\hline 8560 & $3.25(0.98)$ & $2.73-3.71$ & $2.55(0.96)$ & $2.05-3.02$ & $1.21 \mathrm{E}-09$ & $1.90 \mathrm{E}-09$ \\
\hline 8986 & $3.44(1.14)$ & $2.74-3.88$ & $2.86(1.06)$ & $2.23-3.29$ & $2.24 \mathrm{E}-06$ & $2.67 \mathrm{E}-06$ \\
\hline 9626 & $4.54(0.57)$ & $4.25-4.82$ & $4.25(0.97)$ & $3.71-4.68$ & $9.29 \mathrm{E}-05$ & $1.01 \mathrm{E}-04$ \\
\hline 13719 & $0.45(1.76)$ & $-0.51-1.25$ & $2.18(1.22)$ & $1.40-2.62$ & $5.88 \mathrm{E}-15$ & $2.10 \mathrm{E}-14$ \\
\hline 13765 & $4.42(1.19)$ & $3.77-4.96$ & $5.33(1.04)$ & $4.75-5.79$ & $1.08 \mathrm{E}-11$ & $2.25 \mathrm{E}-11$ \\
\hline 13886 & $3.84(1.39)$ & $2.86-4.25$ & $5.43(0.77)$ & $4.89-5.66$ & $1.34 \mathrm{E}-22$ & $1.67 \mathrm{E}-21$ \\
\hline 14049 & $3.71(1.78)$ & $2.64-4.42$ & $4.61(0.96)$ & $4.00-4.96$ & $3.29 \mathrm{E}-11$ & $6.33 \mathrm{E}-11$ \\
\hline 14095 & $1.73(1.02)$ & $1.11-2.13$ & $2.51(0.93)$ & $2.05-2.98$ & $1.31 \mathrm{E}-12$ & $3.28 \mathrm{E}-12$ \\
\hline 14102 & $1.51(0.93)$ & $1.11-2.04$ & $2.55(0.91)$ & $2.06-2.97$ & $8.79 \mathrm{E}-14$ & $2.44 \mathrm{E}-13$ \\
\hline 15123 & $3.68(1.76)$ & $2.85-4.61$ & $1.63(1.35)$ & $1.07-2.42$ & $1.06 \mathrm{E}-17$ & $4.43 \mathrm{E}-17$ \\
\hline 15867 & $4.96(1.29)$ & $4.25-5.54$ & $2.55(1.41)$ & $1.76-3.18$ & $8.81 \mathrm{E}-25$ & $2.20 \mathrm{E}-23$ \\
\hline 28091 & $3.27(0.90)$ & $2.81-3.71$ & $2.44(0.69)$ & $2.18-2.88$ & $2.87 \mathrm{E}-10$ & $5.13 \mathrm{E}-10$ \\
\hline 28232 & $1.70(1.05)$ & $1.14-2.18$ & $3.91(1.49)$ & $2.76-4.25$ & $8.58 \mathrm{E}-21$ & $5.36 \mathrm{E}-20$ \\
\hline
\end{tabular}

*: Adjusted p-value: raw p-value was adjusted for multiple hypothesis testing using a Benjamini-Hochberg correction. 
Table S3. Intensities of feature peaks in the test cohort.

\begin{tabular}{|c|c|c|c|c|c|c|}
\hline \multirow{2}{*}{$m / z$} & \multicolumn{2}{|c|}{ COVID-19 group } & \multicolumn{2}{|c|}{ Control group } & \multirow{2}{*}{ Raw p-value } & \multirow{2}{*}{$\begin{array}{l}\text { Adjusted } \\
\text { p-value* }\end{array}$} \\
\hline & Median (IQR) & $Q_{1}-Q_{3}$ & Median (IQR) & $Q_{1}-Q_{3}$ & & \\
\hline 5158 & $2.29(1.35)$ & $1.61-2.96$ & $1.50(1.17)$ & $0.78-1.94$ & $6.26 \mathrm{E}-05$ & $9.78 \mathrm{E}-05$ \\
\hline 5366 & $2.88(0.66)$ & $2.44-3.10$ & $2.53(0.78)$ & $2.05-2.83$ & $2.33 \mathrm{E}-03$ & $2.91 \mathrm{E}-03$ \\
\hline 5893 & $1.96(0.78)$ & $1.51-2.30$ & $1.16(2.00)$ & $\begin{array}{l}-0.38- \\
1.62\end{array}$ & $1.25 \mathrm{E}-05$ & 2.09E-05 \\
\hline 6357 & $2.10(0.67)$ & $1.73-2.41$ & $2.50(0.94)$ & $1.99-2.94$ & $1.11 \mathrm{E}-02$ & $1.26 \mathrm{E}-02$ \\
\hline 6654 & $3.55(1.05)$ & $2.91-3.95$ & $4.54(1.01)$ & $4.00-5.01$ & $1.89 \mathrm{E}-09$ & 7.57E-09 \\
\hline 6939 & $3.48(1.32)$ & $2.72-4.04$ & $4.61(0.94)$ & $4.02-4.96$ & 2.61E-08 & 5.93E-08 \\
\hline 7364 & $1.93(0.61)$ & $1.59-2.20$ & $1.65(0.88)$ & $1.07-1.95$ & $4.55 \mathrm{E}-03$ & $5.41 \mathrm{E}-03$ \\
\hline 7614 & $4.15(1.24)$ & $3.50-4.75$ & $3.46(1.38)$ & $2.60-3.98$ & $8.11 \mathrm{E}-05$ & $1.19 \mathrm{E}-04$ \\
\hline 8034 & $2.70(0.89)$ & $2.31-3.19$ & $1.68(0.81)$ & $1.20-2.01$ & $1.53 \mathrm{E}-12$ & $1.92 \mathrm{E}-11$ \\
\hline 8043 & $2.56(1.33)$ & $2.05-3.38$ & $1.23(1.22)$ & $0.49-1.71$ & $2.40 \mathrm{E}-10$ & $1.20 \mathrm{E}-09$ \\
\hline 8226 & $1.20(1.32)$ & $0.47-1.78$ & $1.51(1.09)$ & $1.13-2.22$ & $3.23 \mathrm{E}-02$ & $3.36 \mathrm{E}-02$ \\
\hline 8425 & $2.09(0.85)$ & $1.57-2.42$ & $1.69(0.72)$ & $1.22-1.93$ & $1.78 \mathrm{E}-04$ & $2.48 \mathrm{E}-04$ \\
\hline 8560 & $3.27(0.98)$ & $2.76-3.74$ & $2.58(1.42)$ & $1.67-3.09$ & $3.59 \mathrm{E}-06$ & $6.41 \mathrm{E}-06$ \\
\hline 8986 & $3.25(0.91)$ & $2.77-3.68$ & $3.10(1.17)$ & $2.23-3.40$ & $1.44 \mathrm{E}-01$ & $1.44 \mathrm{E}-01$ \\
\hline 9626 & $4.61(0.68)$ & $4.14-4.82$ & $4.36(0.57)$ & $4.04-4.61$ & 2.34E-02 & $2.54 \mathrm{E}-02$ \\
\hline 13719 & $0.38(2.25)$ & $-0.97-1.29$ & $2.14(1.29)$ & $1.31-2.60$ & $5.41 \mathrm{E}-09$ & $1.36 \mathrm{E}-08$ \\
\hline 13765 & $4.48(1.18)$ & $3.77-4.96$ & $5.43(0.60)$ & $5.05-5.66$ & 2.12E-09 & 7.57E-09 \\
\hline 13886 & $3.88(1.18)$ & $3.02-4.25$ & $5.33(0.84)$ & $4.82-5.66$ & $1.77 \mathrm{E}-14$ & $4.42 \mathrm{E}-13$ \\
\hline 14049 & $4.68(0.97)$ & $4.30-5.28$ & $4.68(0.97)$ & $4.30-5.28$ & 3.94E-08 & $8.21 \mathrm{E}-08$ \\
\hline 14095 & $2.64(0.81)$ & $2 . .22-3.03$ & $2.64(0.81)$ & $2.22-3.03$ & 5.43E-09 & $1.36 \mathrm{E}-08$ \\
\hline 14102 & $2.52(0.90)$ & $1.95-2.85$ & $2.52(0.90)$ & $1.95-2.85$ & 2.67E-09 & 8.34E-09 \\
\hline 15123 & $2.10(1.63)$ & $1.38-3.01$ & $2.10(1.63)$ & $1.38-3.01$ & $1.63 \mathrm{E}-07$ & $3.13 \mathrm{E}-07$ \\
\hline 15867 & $2.82(1.42)$ & $2.21-3.63$ & $2.82(1.42)$ & $2.21-3.63$ & $3.51 \mathrm{E}-11$ & 2.92E-10 \\
\hline 28091 & $2.77(0.93)$ & $2.34-3.27$ & $2.77(0.93)$ & $2.34-3.27$ & $1.66 \mathrm{E}-03$ & 2.19E-03 \\
\hline 28232 & $3.80(2.01)$ & $2.33-4.33$ & $3.80(2.01)$ & $2.33-4.33$ & $1.19 \mathrm{E}-10$ & $7.43 \mathrm{E}-10$ \\
\hline
\end{tabular}

*: Adjusted p-value: raw p-value was adjusted for multiple hypothesis testing using a Benjamini-Hochberg correction. 
Table S4. Annotation of the feature peaks.

\begin{tabular}{|c|c|c|c|c|c|}
\hline $\begin{array}{l}\text { Matched Fea- } \\
\text { ture peak }(\mathbf{m} / \mathbf{z})\end{array}$ & $\begin{array}{l}\text { Gene } \\
\text { Name }\end{array}$ & Protein & $\begin{array}{l}\text { Molecular } \\
\text { Weight }\end{array}$ & $\begin{array}{l}\text { Fold Change } \\
\text { in MALDI* }\end{array}$ & $\begin{array}{l}\text { Fold Change in } \\
\text { LC-MS/MS }\end{array}$ \\
\hline 6939 & UBB & Polyubiquitin-B (12-72) & 6934 & 0.8 & 0.4 \\
\hline 7614 & PF4V1 & Platelet factor 4 variant (37-104) & 7610 & 4.7 & $>100$ \\
\hline 8034 & $\begin{array}{c}\text { IGKV3- } \\
15\end{array}$ & $\begin{array}{c}\text { Immunoglobulin kappa variable } \\
3-15(1-74)\end{array}$ & 8019 & 10.0 & 2.9 \\
\hline 8226 & CFI & Complement factor I (1-69) & 8235 & 0.8 & 0.1 \\
\hline 8986 & RAB7A & Ras-related protein Rab-7a (1-79) & 8980 & 1.4 & 24.5 \\
\hline 9626 & ELANE & Neutrophil elastase (1-91) & 9618 & 2.4 & 2.7 \\
\hline 13719 & $\mathrm{~B} 2 \mathrm{M}$ & Beta-2-microglobulin & 13715 & 0.4 & 0.6 \\
\hline 13765 & TTR & Transthyretin (21-147) & 13761 & 0.6 & 0.9 \\
\hline 13886 & PPBP & Platelet basic protein & 13894 & 0.4 & 0.5 \\
\hline 14049 & DUSP14 & $\begin{array}{l}\text { Dual specificity protein phospha- } \\
\text { tase } 14 \text { (76-198) }\end{array}$ & 14057 & 0.5 & 0.2 \\
\hline 14095 & $\mathrm{H} 2 \mathrm{AC} 18$ & Histone $\mathrm{H} 2 \mathrm{~A}$ type $2-\mathrm{A}$ & 14095 & 0.5 & 0.2 \\
\hline 14102 & H2AC6 & Histone $\mathrm{H} 2 \mathrm{~A}$ type $1-\mathrm{C}$ & 14105 & 0.5 & 0.5 \\
\hline 15123 & HBA1 & $\begin{array}{l}\text { Hemoglobin subunit alpha (2- } \\
142)\end{array}$ & 15126 & 16.1 & 5.9 \\
\hline 15867 & $\mathrm{HBB}$ & Hemoglobin subunit beta (2-147) & 15867 & 12.2 & 3.4 \\
\hline 28091 & WRAP73 & $\begin{array}{l}\text { WD repeat-containing protein } \\
\text { WRAP73 (204-460) }\end{array}$ & 28116 & 1.3 & 16.8 \\
\hline
\end{tabular}

*: The fold change was calculated as a ratio (COVID-19/non-COVID-19) of the peak intensity in MALDI-TOF or protein quantity in LC-MS. 


\section{REFERENCES}

1. Gibb, S.; Strimmer, K., MALDIquant: a versatile R package for the analysis of mass spectrometry data. Bioinformatics 2012, 28 (17), 2270-2271.

2. Steinier, J.; Termonia, Y.; Deltour, J., Smoothing and differentiation of data by simplified least square procedure. Anal. Chem. 1972, 44 (11), 1906-1909.

3. Ryan, C. G.; Clayton, E.; Griffin, W. L.; Sie, S. H.; Cousens, D. R., SNIP, a statisticssensitive background treatment for the quantitative-analysis of pixe spectra in geoscience applications. Nucl. Instrum. Meth. B 1988, 34 (3), 396-402.

4. Bolstad, B. M.; Irizarry, R. A.; Astrand, M.; Speed, T. P., A comparison of normalization methods for high density oligonucleotide array data based on variance and bias. Bioinformatics 2003, $19(2), 185-193$.

5. Chong, J.; Wishart, D. S.; Xia, J., Using MetaboAnalyst 4.0 for comprehensive and integrative metabolomics data analysis. Curr. Protoc. Bioinformatics 2019, 68 (1), e86.

6. Tibshirani, R., Regression shrinkage selection via the LASSO. J. R. Stat. Soc. Ser. B-Stat. Methodol. 2011, 73, 273-282.

7. $\mathrm{Xu}, \mathrm{R} . \mathrm{H}$. et al., Circulating tumour DNA methylation markers for diagnosis and prognosis of hepatocellular carcinoma. Nat. Mater. 2017, 16 (11), 1155-1161.

8. $\quad$ Friedman, J.; Hastie, T.; Tibshirani, R., Regularization paths for generalized linear models via coordinate descent. J. Stat. Softw. 2010, 33 (1), 1-22.

9. Guyon, I.; Weston, J.; Barnhill, S.; Vapnik, V., Gene selection for cancer classification using support vector machines. MLear 2002, 46 (1-3), 389-422.

10. Fan, R.-E.; Chang, K.-W.; Hsieh, C.-J.; Wang, X.-R.; Lin, C.-J., LIBLINEAR: A library for large linear classification. J. Mach. Lear. Res. 2008, 9, 1871-1874.

11. Platt, J. C., Probabilistic outputs for support vector machines and comparisons to regularized likelihood methods. MIT Press: 1999.

12. Chan, T. F.; Golub, G. H.; LeVeque, R. J. In Updating formulae and a pairwise algorithm for computing sample variances, COMPSTAT 1982 5th Symposium held at Toulouse 1982, Springer: 1982; pp 30-41.

13. Breiman, L.; Friedman, J.; Stone, C. G.; Olshen, R. A., Classification and regression trees. CRC: 1984.

14. Breiman, L., Random forests. MLear 2001, 45 (1), 5-32.

15. Friedman, J. H., Greedy function approximation: A gradient boosting machine. Ann. Stat. 2001, 29 (5), 1189-1232.

16. Friedman, J., Stochastic gradient boosting. Department of Statistics, Stanford University: 1999.

17. Zhu, J.; Zou, H.; Rosset, S.; Hastie, T., Multi-class AdaBoost. Stat. Interface 2009, 2 (3), 349-360.

18. Wang, H., Nearest neighbours without $k$ : A classification formalism based on probability. Faculty of Informatics, University of Ulster: 2002.

19. Sokolova, M.; Japkowicz, N.; Szpakowicz, S., Beyond accuracy, F-Score and ROC: A family of discriminant measures for performance evaluation. In Ai 2006: Advances in Artificial Intelligence, Proceedings, Sattar, A.; Kang, B. H., Eds. 2006; Vol. 4304, pp 1015-1021.

20. Zhou, Y.; Zhou, B.; Pache, L.; Chang, M.; Khodabakhshi, A. H.; Tanaseichuk, O.; Benner, C.; Chanda, S. K., Metascape provides a biologist-oriented resource for the analysis of systems-level datasets. Nat. Commun. 2019, 10, 15123. 